\title{
Mathematical modelling of bacterial resistance to multiple antibiotics and immune system response
}

\author{
Bahatdin Daşbaşı ${ }^{1 *}$ and IIlhan Öztürk ${ }^{2}$
}

${ }^{*}$ Correspondence:

dasbasibahatdin@gmail.com

${ }^{1}$ Department of Computer

Technologies, Kangal

Vocational High-Schools,

Cumhuriyet University,

58840 Sivas, Turkey

Full list of author information

is available at the end of the article

\begin{abstract}
Resistance of developed bacteria to antibiotic treatment is a very important issue, because introduction of any new antibiotic is after a little while followed by the formation of resistant bacterial isolates in the clinic. The significant increase in clinical resistance to antibiotics is a troubling situation especially in nosocomial infections, where already defenseless patients can be unsuccessful to respond to treatment, causing even greater health issue. Nosocomial infections can be identified as those happening within 2 days of hospital acceptance, 3 days of discharge or 1 month of an operation. They influence 1 out of 10 patients admitted to hospital. Annually, this outcomes in 5000 deaths only in UK with a cost to the National Health Service of a billion pounds. Despite these problems, antibiotic therapy is still the most common method used to treat bacterial infections. On the other hand, it is often mentioned that immune system plays a major role in the progress of infections. In this context, we proposed a mathematical model defining population dynamics of both the specific immune cells produced according to the properties of bacteria by host and the bacteria exposed to multiple antibiotics synchronically, presuming that resistance is gained through mutations due to exposure to antibiotic. Qualitative analysis found out infection-free equilibrium point and other equilibrium points where resistant bacteria and immune system cells exist, only resistant bacteria exists and sensitive bacteria, resistant bacteria and immune system cells exist. As a result of this analysis, our model highlights the fact that when an individual's immune system weakens, he/she suffers more from the bacterial infections which are believed to have been confined or terminated. Also, these results was supported by numerical simulations.
\end{abstract}

Keywords: Ordinary differential equations systems, Equilibrium points, Immune system, Bacterial resistance, Antibiotics

Mathematics Subject Classification: 34K20, 92C50, 92D25

\section{Background}

Infections have been the leading cause of most diseases in the history of mankind (Mondragón et al. 2014). Especially bacterial infections are more prevailing among these. The most common procedure known to fight bacterial infection is through antibiotic therapy applied to individuals. The expression of resistance to antimicrobial agents in

\section{SpringerOpen}

๑ 2016 Daşbaşı and Öztürk. This article is distributed under the terms of the Creative Commons Attribution 4.0 International License (http://creativecommons.org/licenses/by/4.0/, which permits unrestricted use, distribution, and reproduction in any medium, provided you give appropriate credit to the original author(s) and the source, provide a link to the Creative Commons license, and indicate if changes were made. 
this therapy is both the logical and indispensable outcome of using these agents to treat human infections (Mahmoud and Rice 1999; Ternent et al. 2014).

Resistance developed by the bacteria against antibiotics is described as the talent of bacteria to resist the effects of antibiotics generated either to eradicate or control them (Arya 2007). The release of each new class of antibiotics for treatment, shortly after, has been followed by the emergence of new strains of bacteria which are resistant to this class (Butler and Buss 2006; Clatworthy et al. 2007; Lewis 2013). In this respect, developing new treatment strategies for bacterial infections is very important (Mondragón et al. 2014).

According to its properties, antibiotics has the bacteriostatic action to stop the growth of bacteria and bactericidal action to wipe out the bacteria. However, the distinction between these properties is not explicit, as it depends on the drug concentration used and the type and the growth stage of bacteria (Zhang 2009). Hence, multiple antibiotic therapy is a more appropriate form of treatment.

In fact, the bacterial infection is a complicated process for both the infectious bacteria and the host (Carvalho et al. 2012). It is suggested that a significant role in the progress of infections is basically played by the immune system (Linares and Martinez 2005). The immune system is expressed as a system of biological structures and processes in an organism protecting the body from likely harmful substances via recognizing and responding to antigens (Alberts et al. 2002). In this context, the reactions of different hosts against the same infection can be different due to immune system's response given by host.

In light of the above, dynamics of relations among antibiotics, immune system's cells and bacteria are important to understand the nature of the infection.

Mathematical models are one of the significant tools used in both analyzing the spread of infectious diseases in a population of individuals (Hethcote 2000; Singer 1984), and predicting the timing and expansion of infection and possible reinfection processes in an individual (Mohtashemi and Levins 2001). While the former is usually used for planning, prevention and control scenarios, the latter can be active in therapy/intervention programs for treating the individuals exposed to the particular pathogen. In this respect, understanding and anticipating the time of occurrence and magnitude of the maximum load of the bacteria and immune system cells by mathematical modelling can be crucial in selecting effective intervention strategies (Whitman and Ashrafiuon 2006).

Consequently, results on reproduction of sensitive and resistant bacteria to antibiotics are obtained in Austin et al. (1997), Bonten et al (2001), Esteva et al. (2011), Wiesch et al. (2011), Zhang (2009); definitions of factors responsible for resistance prevalence are studied in Austin and Anderson (1999), Linares and Martinez (2005), Opatowski et al. (2011), Rodrigues et al. (2007); bacteria behavior under different antibiotic treatments is examined in Alavez et al. (2006), Bonhoeffer et al. (1997), Bootsma et al. (2012), D’Agata et al. (2007), Sun et al. (2010); optimization results and design of control measures are investigated in Bonten et al. (2001), Haber et al. (2010), Massad et al. (2008), Sotto and Lavigne (2012); biological cost and persistence of antibiotic resistance are analyzed in Andersson et al. (2001), Andersson and Levin (1999), Antia et al. (1996), Johnson and Levin (2013), Mondragón et al. (2014); dynamics between pathogens and immune response are given in André and Gandon (2006), Carvalho et al. (2012), 
D'Onofrio (2005), Gilchrist and Coombs (2006), Gilchrist and Sasaki (2002), Kostova (2007), Mohtashemi and Levins (2001), Nowak and May (2000), Whitman and Ashrafiuon (2006), respectively.

In this study, we have constructed a continuous time model considering the immune response of the host and the basic mechanisms of bacterial resistance to antibiotics. Our aim is to find specific parameters determining the change in the concentrations of the immune system's cells produced in host to fight these and the sensitive sub-populations and resistant sub-populations that has either arisen through random mutation and clonal enlargement or through cross-contamination in a special infection and under a appropriate treatment regimen.

\section{Model formulation}

When the emergence of resistant bacteria to antibiotic has modelled, there are the two basic aspects generally considered. These are within-host models and within-hospital compartmental models. Within-hospital compartmental models are generally SIR models in epidemiology, considering especially how infections will expand throughout the hospital (Ternent et al. 2014). These models are useful to develop strategies to prevent the spread of resistant individuals to antibiotic in hospital.

Current mathematical models focusing on the fact that it is within the host that resistance develops, aim to study how antibiotic treatment strategies can affect, and in addition, how the occurrence of antibiotic resistance can be prevented (Alberts et al. 2002; Hethcote 2000). In such models, the effect of immune cells generated by the host under the pressure of the bacteria are frequently either neglected or assumed to be at a constant rate. We built up the mathematical model including cell-mediated immune response. In addition, treatment regimens involving multiple antibiotic have been used in most bacterial infections due to bacterial resistant. In this sense, besides the interaction of bacterial-immune response, to investigate the effects of multiple drug therapy is biological more meaningful.

We analyzed the concentrations of the immune cells and bacteria in an individual receiving a cocktail of multi-drug treatment against bacteria via mathematical modelling. Let us denote by $S(t)$ and $R(t)$ the population sizes of sensitive and resistant bacteria against multiple antibiotics at time $t$, respectively, by $B(t)$ population sizes of immune cells at time $t$, and by $A_{i}(t)$ the concentration of the $i$-th antibiotic, for $i=1,2, \ldots, n$ at time $t$. The parameters used in the model are as follows.

We assume that bacteria follow a logistic growth with carrying capacity $T$. Let $\beta_{S}$ and $(1-c) \beta_{S}$ the birth rate of sensitive and resistant bacteria, respectively. Specific mutations arising from resistance to chemical control frequently have an inherent fitness cost which may be resulted through reduced reproductive capacity or competitive ability (Alavez et al. 2006). We evaluate fitness cost as a reduction on the reproduction rate of the resistant strain, therefore $0<c<1$. Likewise, we have supposed that using a logistic style term, immune cells are recruited to the region of infection at a rate $k$ and carrying capacity of these is as $\omega$ fold of amount of present bacteria. This is biologically very significant in terms of proliferation of specific immune cells. Also, these interacts can be expressed as a generalised model of a local bacterial infection, such as a urinary tract, tuberculosis or wound infection. 
During the administration of the $i$ th antibiotic, a number of resistant bacteria to it can be showed up due to mutations of exposed sensitive bacteria to such antibiotic, we model this situation by the term $\overline{\alpha_{i}} A_{i} S$ where $\overline{\alpha_{i}}$ is the mutation rate of sensitive bacteria due to exposure to $i$ th antibiotic. Sensitive and resistant bacteria have per capita death rates by response of immune cells and this rates is $\lambda$. Sensitive bacteria also die due to the action of the antibiotics, and we have assumed that this situation in model is by the term $\overline{d_{i}} A_{i} S$, where $\overline{d_{i}}$ is the death rate of sensitive bacteria due to exposure to $i$-th antibiotic. Lastly, the $i$-th antibiotic concentration is supplied at a constant rate $\delta_{i}$, and is taken up at a constant per capita rate $\mu_{i}$. Under the assumptions a fore mentioned, we obtain the following system of $(n+3)$ ODE's:

$$
\begin{aligned}
\frac{d S}{d t} & =\beta_{S} S\left(1-\frac{S+R}{T}\right)-\lambda S B-S \sum_{i=1}^{n} \overline{\alpha_{i}} A_{i}-S \sum_{i=1}^{n} \overline{d_{i}} A_{i} \\
\frac{d R}{d t} & =(1-c) \beta_{S} R\left(1-\frac{S+R}{T}\right)-\lambda R B+S \sum_{i=1}^{n} \overline{\alpha_{i}} A_{i} \\
\frac{d B}{d t} & =k B\left(1-\frac{B}{\omega(S+R)}\right) \\
\frac{d A_{i}}{d t} & =\delta_{i}-\mu_{i} A_{i}, \quad i=1,2, \ldots, n .
\end{aligned}
$$

where

$$
\beta_{S}, c, \lambda, T, k, \omega, \overline{\alpha_{i}}, \overline{d_{i}}, \delta_{i}, \mu_{i}>0 \quad \text { for } i=1,2, \ldots, n
$$

To reduce the number of parameters, the variables are changed as follows;

$$
s=\frac{S}{T}, r=\frac{R}{T}, b=\frac{B}{\omega T}, a_{i}=\frac{A_{i}}{\frac{\delta_{i}}{\mu_{i}}}
$$

With the new variables, the normalized system is given as;

$$
\begin{aligned}
& \frac{d s}{d t}=\beta_{S} s(1-(s+r))-\eta s b-s \sum_{i=1}^{n}\left(\alpha_{i}+d_{i}\right) a_{i} \\
& \frac{d r}{d t}=\beta_{R} r(1-(s+r))-\eta r b+s \sum_{i=1}^{n} \alpha_{i} a_{i} \\
& \frac{d b}{d t}=k b\left(1-\frac{b}{s+r}\right) \\
& \frac{d a_{i}}{d t}=\mu_{i}\left(1-a_{i}\right) \quad \text { for } i=1,2, \ldots, n
\end{aligned}
$$

where $\alpha_{i}=\bar{\alpha}_{i}\left(\frac{\delta_{i}}{\mu_{i}}\right), d_{i}=\bar{d}_{i}\left(\frac{\delta_{i}}{\mu_{i}}\right), \beta_{R}=(1-c) \beta_{S}$ and $\eta=\lambda \omega T$. The biologically studied region is given by the set

$$
\Omega=\left\{\left(s, r, b, a_{1}, \ldots, a_{n}\right) \in R^{n+3}: 0 \leq s, r, 0 \leq b \leq s+r \leq 1,0 \leq a_{i} \leq 1, i=1, \ldots, n\right\} .
$$


The following proposition ensures that system (3) is well posed in the sense that the solutions with positive initial conditions started in $\Omega$ remain in this region for all $t \geq 0$, and so, this solutions of system (3) have biological meaning.

Proposition 1 The region $\Omega$ defined in (4) is positively invariant with respect to the system (3).

Proof By adding the first two equations of the system (3),

$$
\frac{d s}{d t}+\frac{d r}{d t}=\left(\beta_{S} s+\beta_{R} r\right)(1-(s+r))-\eta b(s+r)-s \sum_{i=1}^{n} d_{i} a_{i}
$$

is obtained. Considering the region $\Omega$, we have the following inequality;

$$
\frac{d(s+r)}{d t} \leq \beta_{S}(s+r)(1-(s+r)) \text {. }
$$

By the solution according to $(s+r)$ of inequality (6), it follows that $0 \leq s+r \leq 1$ for all $t \geq 0$. Furthermore, the solutions of the last $n$ equations of system (3) are

$$
a_{i}(t)=1+\left(-1+a_{i}(0)\right) e^{-\mu_{i} t} \quad \text { for } i=1,2, \ldots, n .
$$

where initial conditions satisfy $0 \leq a_{i}(0) \leq 1$ for $i=1,2, \ldots, n$. Lastly, let $0 \leq s+r=u$ (constant) $\leq 1$. Then the solution of third equation in system (3) is

$$
b=\frac{u}{1+e^{-k t-b(0)}}
$$

where initial conditions satisfy $0<b(0) \leq s(0)+r(0)$. From (8), it is obtained that $0 \leq b \leq s+r \leq 1$.

Hence, the vector field of system (3) restricted to the boundary of $\Omega$ does not include a point at the exterior of it. In this context, solutions starting in $\Omega^{+}$remain in the region $\Omega$ for all $t \geq 0$.

\section{Qualitative analysis of system (3)}

We have examined the existence and stability of equilibria of system (3).

\section{Proposition 2 Let}

$$
\frac{\beta_{S}-\sum_{i=1}^{n}\left(\alpha_{i}+d_{i}\right)}{\beta_{S}+\eta}=A, \quad \frac{\beta_{R}}{\beta_{R}+\eta}=B, \quad \frac{\sum_{i=1}^{n} \alpha_{i}}{\beta_{R}+\eta}=C .
$$

We accept that the general expressions of the system's equilibria show as $E_{j}=\left(\bar{s}, \bar{r}, \bar{b}, \overline{a_{i}}\right)$ for $i=1,2, \ldots, n$ and $j=0,1,2,3$. Then, system (3) always has $E_{0}=(0,0,0,1,1, \ldots, 1)$ (namely, the infection-free equilibrium point), $E_{1}=(0,1,0,1,1, \ldots, 1), E_{2}=(0, B, B, 1,1$, ..., 1) contained in $\Omega$. When $A>B$, in addition to $E_{0}, E_{1}$ and $E_{2}$, there exists a fourth the equilibrium point, $E_{3}=\left(A \frac{A-B}{A-B+C}, A \frac{C}{A-B+C}, A, 1,1, \ldots, 1\right)$, in $\Omega$. 
Proof In (9), it is clear that

$$
B, C>0
$$

The equilibria of system (3) are given by the solutions of the system of following algebraic equations;

$$
\begin{aligned}
& \beta_{S}(1-(s+r))-\eta s b-s \sum_{i=1}^{n}\left(\alpha_{i}+d_{i}\right) a_{i}=0 \\
& \beta_{R} r(1-(s+r))-\eta r b+s \sum_{i=1}^{n} \alpha_{i} a_{i}=0 \\
& k b\left(1-\frac{b}{s+r}\right)=0 \\
& \mu_{i}\left(1-a_{i}\right)=0 \text { for } i=1,2, \ldots, n .
\end{aligned}
$$

From the last $n$ equation of system (11), we have $a_{i}=1$ for $i=1,2, \ldots, n$. Consequently, the system (11) turns into following system;

$$
\begin{aligned}
& \beta_{S} s(1-(s+r))-\eta s b-s \sum_{i=1}^{n}\left(\alpha_{i}+d_{i}\right)=0 \\
& \beta_{R} r(1-(s+r))-\eta r b+s \sum_{i=1}^{n} \alpha_{i}=0 . \\
& k b\left(1-\frac{b}{s+r}\right)=0 .
\end{aligned}
$$

From (12), it is obtained that either $\bar{b}=0$ or $\bar{b}=\bar{s}+\bar{r}$.

- When $\bar{b}=0$, we have that the equilibrium points are $E_{0}=(0,0,0,1, \ldots, 1)$, $E_{1}=(0,1,0,1, \ldots, 1)$ and

$$
\begin{aligned}
E^{l}= & \left(-\frac{\beta_{S}-\sum_{i=1}^{n}\left(\alpha_{i}+d_{i}\right)}{\beta_{S} \sum_{i=1}^{n} \alpha_{i}-\beta_{R} \sum_{i=1}^{n}\left(\alpha_{i}+d_{i}\right)} \frac{\beta_{R}}{\beta_{S}} \sum_{i=1}^{n}\left(\alpha_{i}+d_{i}\right),\right. \\
& \left.\frac{\beta_{S}-\sum_{i=1}^{n}\left(\alpha_{i}+d_{i}\right)}{\beta_{S} \sum_{i=1}^{n} \alpha_{i}-\beta_{R} \sum_{i=1}^{n}\left(\alpha_{i}+d_{i}\right)} \sum_{i=1}^{n} \alpha_{i}, 0,1, \ldots, 1\right)
\end{aligned}
$$

Although the equilibrium points $E_{0}$ and $E_{1}$ always exist in $\Omega$, the equilibrium point $E^{I}$ where signs of $\bar{s}$ and $\bar{r}$ are opposite, is biologically meaningless. Therefore, $E^{I}$ is not in $\Omega$.

- In case of $\bar{b}=\bar{s}+\bar{r}$, it is obtained the equilibrium points as following;

- $E_{2}=\left(0, \frac{\beta_{R}}{\beta_{R}+\eta}, \frac{\beta_{R}}{\beta_{R}+\eta}, 1, \ldots, 1\right)$ and

$$
E_{3}=\left(\bar{b} \frac{\beta_{R}-\bar{b}\left(\beta_{R}+\eta\right)}{\left(\beta_{R}-\bar{b}\left(\beta_{R}+\eta\right)\right)-\sum_{i=1}^{n} \alpha_{i}},-\bar{b} \frac{\sum_{i=1}^{n} \alpha_{i}}{\left(\beta_{R}-\bar{b}\left(\beta_{R}+\eta\right)\right)-\sum_{i=1}^{n} \alpha_{i}}, \bar{b}=\frac{\beta_{S}-\sum_{i=1}^{n}\left(\alpha_{i}+d_{i}\right)}{\left(\beta_{S}+\eta\right)}, 1, \ldots, 1 .\right)
$$

Equilibrium point $E_{2}$ always exists in $\Omega$. In addition that, the equilibrium point $E_{3}$ is in $\Omega$, when $\frac{\beta_{S}-\sum_{i=1}^{n}\left(\alpha_{i}+d_{i}\right)}{\beta_{S}+\eta}>\frac{\beta_{R}}{\beta_{R}+\eta}$. 
Taking (9) into account, we have that equilibria of system (3) in $\Omega$ are

$$
\begin{aligned}
& E_{0}=(0,0,0,1, \ldots, 1) \\
& E_{1}=(0,1,0,1, \ldots, 1) \\
& E_{2}=(0, B, B, 1, \ldots, 1) \\
& E_{3}=\left(A \frac{A-B}{A-B+C}, A \frac{C}{A-B+C}, A, 1, \ldots, 1\right), \quad \text { when } A>B .
\end{aligned}
$$

Theorem 3 (Routh-Hurwitz Criteria) Given the polynomial,

$$
P(\lambda)=\lambda^{n}+a_{1} \lambda^{n-1}+\cdots+a_{n-1} \lambda+a_{n},
$$

where the coefficients $a_{i}$ for $i=1, \ldots, n$ are real constants, define the $n$ Hurwitz matrices using the coefficients $a_{i}$ of the characteristic polynomial:

$$
H_{1}=\left(a_{1}\right), H_{2}=\left(\begin{array}{ll}
a_{1} & 1 \\
a_{3} & a_{2}
\end{array}\right), H_{3}=\left(\begin{array}{lll}
a_{1} & 1 & 0 \\
a_{3} & a_{2} & a_{1} \\
a_{5} & a_{4} & a_{3}
\end{array}\right), \ldots, H_{n}=\left(\begin{array}{cccccc}
a_{1} & 1 & 0 & 0 & \cdots & 0 \\
a_{3} & a_{2} & a_{1} & 1 & \cdots & 0 \\
a_{5} & a_{4} & a_{3} & a_{1} & \cdots & 0 \\
\vdots & \vdots & \vdots & \vdots & \ddots & \vdots \\
0 & 0 & 0 & 0 & \cdots & a
\end{array}\right)
$$

where $a_{j}=0$ if $j>n$. All of the roots of polynomial $P(\lambda)$ are negative or have negative real parts, if and only if the determinants of all Hurwitz matrices are positive: $\operatorname{det} H_{j}>0, j=1,2, \ldots, n$. For polynomial of degree $n=2,3,4$ and 5 , the Routh-Hurwitz criteria are summarized.

$$
\begin{aligned}
n=2 & : a_{1}, a_{2}>0 \\
n= & 3: a_{1}, a_{3}>0 \text { and } a_{1} a_{2}>a_{3}, \\
n= & 4: a_{1}, a_{3}, a_{4}>0 \text { and } a_{1} a_{2} a_{3}>a_{3}^{2}+a_{1}^{2} a_{4}, \\
n= & 5: a_{1}, a_{2}, a_{3}, a_{4}, a_{5}>0, a_{1} a_{2} a_{3}>a_{3}^{2}+a_{1}^{2} a_{4} \text { and } \\
& \quad\left(a_{1} a_{4}-a_{5}\right)\left(a_{1} a_{2} a_{3}-a_{3}^{2}-a_{1}^{2} a_{4}\right)>a_{5}\left(a_{1} a_{2}-a_{3}\right)^{2}+a_{1} a_{5}^{2} .
\end{aligned}
$$

This criteria has given necessary and sufficient conditions for all of the roots of the characteristic polynomial (with real coefficients) to lie in the left half of the complex plane (Allen 2007).

Theorem 4 Suppose $\frac{d X}{d t}=F(X)$ is a nonlinear first-order autonomous system with an equilibrium $\bar{X}$. Denote the Jacobian matrix of $F$ evaluated at $\bar{X}$ as $J(\bar{X})$. If the characteristic equation of the Jacobian matrix $J(\bar{X})$,

$$
\lambda^{n}+a_{1} \lambda^{n-1}+a_{2} \lambda^{n-2}+\cdots+a_{n-1} \lambda+a_{n}=0,
$$

satisfies the conditions of the Routh-Hurwitz criteria in Theorem 3, that is, the determinants of all of the Hurwitz matrices are positive, $\operatorname{det}\left(H_{j}\right)>0, j=1,2, \ldots, n$, then the equilibrium $\bar{X}$ is locally asimptotically stable. If $\operatorname{det}\left(H_{j}\right)<0$, for some $j=1,2, \ldots, n$, then the equilibrium $\bar{X}$ is unstable (Allen 2007).

The following proposition is shown conditions that equilibrium points in the Proposition 2 are locally asimptotically stability (LAS). 
Proposition 4 The equilibrium points of system (3) in $\Omega$ satisfy

(i) $\quad E_{0}$ and $E_{1}$ are unstable points.

(ii) If $A<B$, then $E_{2}$ is LAS.

(iii) Let $B<A$, then $E_{3}$ is $L A S$.

Proof For the stability analysis, the functions of the right side of system (3) are determined as follows;

$$
\begin{aligned}
& \varphi_{1}\left(s, r, b, a_{i}\right)=\beta_{S} s(1-(s+r))-\eta s b-s \sum_{i=1}^{n}\left(\alpha_{i}+d_{i}\right) a_{i} \\
& \varphi_{2}\left(s, r, b, a_{i}\right)=\beta_{R} r(1-(s+r))-\eta r b+s \sum_{i=1}^{n} \alpha_{i} a_{i} \\
& \varphi_{3}\left(s, r, b, a_{i}\right)=k b\left(1-\frac{b}{s+r}\right) \\
& r_{i}\left(s, r, b, a_{i}\right)=\mu_{i}\left(1-a_{i}\right), \quad i=1,2, \ldots, n .
\end{aligned}
$$

That jacobian matrix obtained from the equations in (14) is

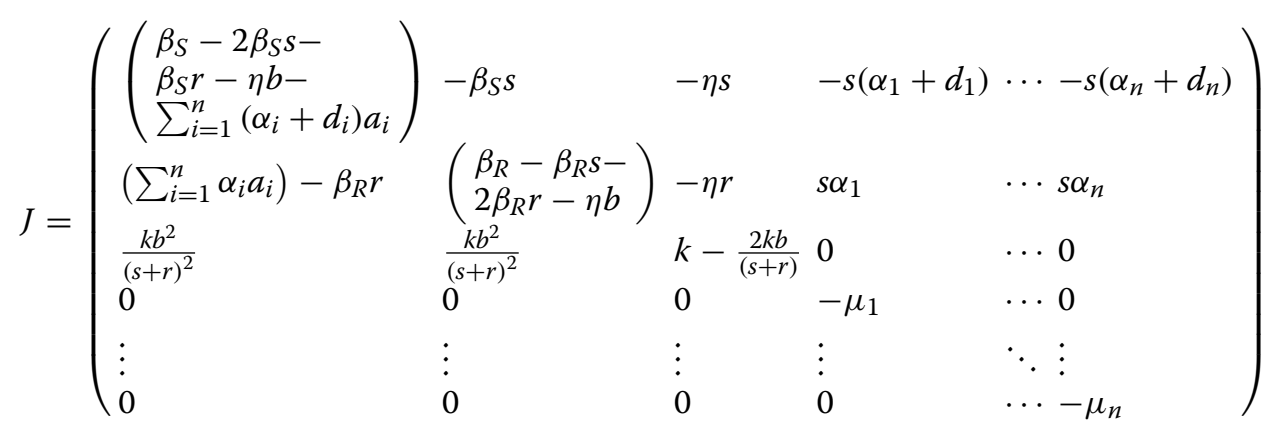

For ease of examination, the $\tau$-th eigenvalue of equilibrium point $E_{k}$ has shown as $\lambda_{k, \tau}$ for $k=0,1,2,3$ and $\tau=1,2, \ldots, n+3, \quad n \in N$.

(i) Some of the eigenvalues evaluated at the equilibrium point $E_{0}$ in $\Omega$ are $\lambda_{0,1}=\beta_{S}-\sum_{i=1}^{n}\left(\alpha_{i}+d_{i}\right)$ and $\lambda_{0,2}=\beta_{R}$. The eigenvalue $\lambda_{0,2}$ is positive, due to (2). According to Theorem 4, the infection-free equilibrium point $E_{0}$ is unstable point for system (3).

In the same way, the eigenvalues for $E_{1}$ in $\Omega$ are that $\lambda_{1,1}=-\sum_{i=1}^{n}\left(\alpha_{i}+d_{i}\right)$, $\lambda_{1,2}=-\beta_{R}, \lambda_{1,3}=k$ and $\lambda_{1, i+3}=-\mu_{i}$ for $i=1,2, \ldots, n$. The eigenvalue $\lambda_{1,3}$ is positive, due to (2). From Theorem 4, $E_{1}$ is unstable point for system (3).

(ii) The jacobian matrix in (15) evaluated at the equilibrium point $E_{2}$ in $\Omega$ is given below

$$
J\left(E_{2}\right)=\left(\begin{array}{llllll}
\beta_{S}-B\left(\beta_{S}+\eta\right)-\sum_{i=1}^{n}\left(\alpha_{i}+d_{i}\right) & 0 & 0 & 0 & \ldots & 0 \\
-\beta_{R} B+\sum_{i=1}^{n} \alpha_{i} & -\beta_{R} B & -\eta B & 0 & \ldots & 0 \\
k & k & -k & 0 & \ldots & 0 \\
0 & 0 & 0 & -\mu_{1} & \ldots & 0 \\
\vdots & \vdots & \vdots & \vdots & \ddots & \vdots \\
0 & 0 & 0 & 0 & \ldots & -\mu_{n}
\end{array}\right)
$$


The eigenvalues of matrix (16) are $\lambda_{2, i+3}=-\mu_{i}$ for $i=1,2, \ldots, n$ and the remain three eigenvalues are found from following matrix;

$$
J^{B\left(E_{2}\right)}=\left(\begin{array}{llc}
\beta_{S}-B\left(\beta_{S}+\eta\right)-\sum_{i=1}^{n}\left(\alpha_{i}+d_{i}\right) & 0 & 0 \\
-\beta_{R} B+\sum_{i=1}^{n} \alpha_{i} & -\beta_{R} B & -\eta B \\
k & k & -k
\end{array}\right)
$$

where matrix $J^{B\left(E_{2}\right)}$ is the block matrix of (16). From (2), it is clear that $R e \lambda_{2, i+3}<0$. Characteristic equation of (17) is

$$
\left[\left(\beta_{S}-B\left(\beta_{S}+\eta\right)-\sum_{i=1}^{n}\left(\alpha_{i}+d_{i}\right)\right)-\lambda\right]\left[\lambda^{2}+\lambda\left(k+\beta_{R} B\right)+k \beta_{R}\right]=0 .
$$

By the solution of (18), these eigenvalues are determined. Hence, it is obtained that

$$
\lambda_{2,1}=\left(\beta_{S}-B\left(\beta_{S}+\eta\right)-\sum_{i=1}^{n}\left(\alpha_{i}+d_{i}\right)\right)
$$

and $\lambda_{2,2}$ and $\lambda_{2,3}$ are gained by solving following equation,

$$
\lambda^{2}+\lambda\left(k+\beta_{R} B\right)+k \beta_{R}=0 .
$$

It can be observed that $\left(k+\beta_{R} B\right), k \beta_{R}>0$, due to (2) and (10). From Theorem 3 $(n=2), \operatorname{Re} \lambda_{2,2}$ and $\operatorname{Re} \lambda_{2,3}<0$. According to Theorem 4, the LAS conditions for $E_{2}$ are provided for the eigenvalues, $\lambda_{2, i+3}, \lambda_{2,2}$ and $\lambda_{2,3}$, exceptionally $\lambda_{2,1}$ in the (19). It is sufficient to examine the sign of $\lambda_{2,1}$. By (9), (19) it can be rewrited as $\lambda_{2,1}=\left(\beta_{S}+\eta\right)(A-B)$. In this respect, $\lambda_{2,1}$ is negative, when $A<B$.

As a result, the equilibrium point $E_{2}$ is LAS for system (3), when $A<B$.

(iii) Let $B<A$. Then the equilibrium point $E_{3}$ is revealed in $\Omega$. The Jacobian matrix for $E_{3}$ is

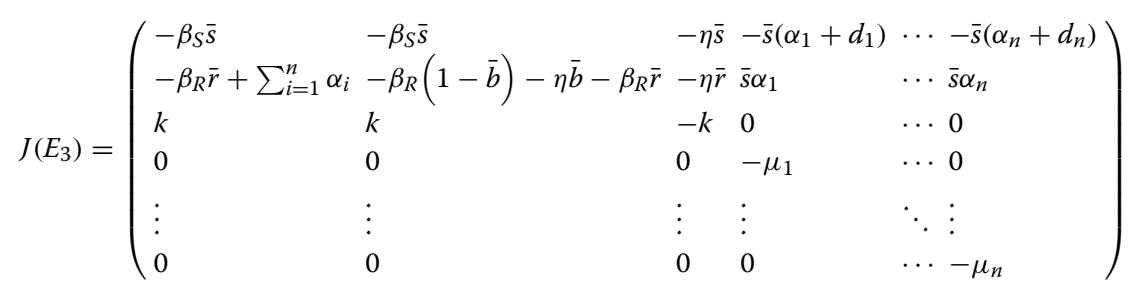

where

$$
E_{3}=\left(A \frac{A-B}{A-B+C}, A \frac{C}{A-B+C}, A, 1,1, \ldots, 1\right)=(\bar{s}, \bar{r}, \bar{b}, 1,1, \ldots, 1) \quad \text { for } A>B .
$$

The eigenvalues obtained from (21) are $\lambda_{3, i+3}=-\mu_{i}$ for $i=1,2, \ldots, n$ and the remaining three eigenvalues, $\lambda_{3,1}, \lambda_{3,2}$ and $\lambda_{3,3}$, are found from following matrix;

$$
J^{B\left(E_{3}\right)}=\left(\begin{array}{llr}
-\beta_{S} \bar{s} & -\beta_{S} \bar{s} & -\eta \bar{s} \\
-\beta_{R} \bar{r}+\sum_{i=1}^{n} \alpha_{i} & \beta_{R}(1-\bar{b})-\eta \bar{b}-\beta_{R} \bar{r} & -\eta \bar{r} \\
k & k & -k
\end{array}\right)
$$

where the matrix $J^{B\left(E_{3}\right)}$ is the block matrix of (21). By (2), it is $\operatorname{Re} \lambda_{3, i+3}<0$. Characteristic equation of matrix (23) is obtained as follows: 
$\lambda^{3}+a_{1} \lambda^{2}+a_{2} \lambda+a_{3}=0$,

where

$$
\begin{aligned}
& a_{1}=\left(\left(\frac{\bar{s}}{\bar{r}} \sum_{i=1}^{n} \alpha_{i}+\beta_{R} \bar{r}+\beta_{S} \bar{s}\right)+k\right) \\
& a_{2}=\left(k\left(\frac{\bar{s}}{\bar{r}} \sum_{i=1}^{n} \alpha_{i}+\beta_{R} \bar{r}+\beta_{S} \bar{s}\right)+\bar{b} \eta k+\beta_{S} \bar{b} \overline{\bar{r}} \sum_{i=1}^{n} \alpha_{i}\right) \\
& a_{3}=k \eta \bar{b} \frac{\bar{s}}{\bar{s}} \sum_{i=1}^{n} \alpha_{i}+k \beta_{S} \bar{b} \overline{\bar{s}} \sum_{i=1}^{n} \alpha_{i} .
\end{aligned}
$$

We have $a_{1}, a_{3}>0$ due to (2) and (22). By (25), we have that

$$
\begin{aligned}
a_{1} a_{2}-a_{3}= & \left(\left(\frac{\bar{s}}{\bar{r}} \sum_{i=1}^{n} \alpha_{i}+\beta_{R} \bar{r}+\beta_{S} \bar{s}\right)+k\right)\left(k\left(\overline{\bar{s}} \sum_{i=1}^{n} \alpha_{i}+\beta_{R} \bar{r}+\beta_{S} \bar{s}\right)+\bar{b} \eta k+\bar{b} \beta_{S} \overline{\bar{s}} \sum_{i=1}^{n} \alpha_{i}\right) \\
& -k \bar{b}\left(\bar{s} \sum_{i=1}^{n} \alpha_{i}\right)\left(\eta+\beta_{S}\right), \\
a_{1} a_{2}-a_{3}= & \left(\frac{\bar{s}}{\bar{r}} \sum_{i=1}^{n} \alpha_{i}\right) \bar{b} \eta k+\left(\frac{\bar{s}}{\bar{r}} \sum_{i=1}^{n} \alpha_{i}\right)\left(\bar{b} \beta_{S}\left(\frac{\bar{s}}{\bar{r}} \sum_{i=1}^{n} \alpha_{i}\right)+k\left(\frac{\bar{s}}{\bar{r}} \sum_{i=1}^{n} \alpha_{i}+\beta_{R} \bar{r}+\beta_{S} \bar{s}\right)\right) \\
& +k \bar{b} \beta_{S}\left(\frac{\bar{s}}{\bar{r}} \sum_{i=1}^{n} \alpha_{i}\right)+k\left(\bar{b} \eta k+k\left(\frac{\bar{s}}{\bar{r}} \sum_{i=1}^{n} \alpha_{i}+\beta_{R} \bar{r}+\beta_{S} \bar{s}\right)\right) \\
& +\left(\beta_{R} \bar{r}+\beta_{S} \bar{s}\right)\left(\bar{b} \eta k+\bar{b} \beta_{S}\left(\frac{\bar{s}}{\bar{r}} \sum_{i=1}^{n} \alpha_{i}\right)+k\left(\frac{\bar{s}}{\overline{\bar{r}}} \sum_{i=1}^{n} \alpha_{i}+\beta_{R} \bar{r}+\beta_{S} \bar{s}\right)\right) \\
& -k \bar{b}\left(\bar{s} \sum_{i=1}^{n} \alpha_{i}\right)\left(\eta+\beta_{S}\right)
\end{aligned}
$$

and so,

$$
\begin{aligned}
a_{1} a_{2}-a_{3}= & \left(\frac{\bar{s}}{\bar{r}} \sum_{i=1}^{n} \alpha_{i}\right)\left(\bar{b} \beta_{S}\left(\frac{\bar{s}}{\bar{r}} \sum_{i=1}^{n} \alpha_{i}\right)+k\left(\frac{\bar{s}}{\bar{r}} \sum_{i=1}^{n} \alpha_{i}+\beta_{R} \bar{r}+\beta_{S} \bar{s}\right)\right) \\
& +k\left(\bar{b} \eta k+k\left(\frac{\bar{s}}{\overline{\bar{r}}} \sum_{i=1}^{n} \alpha_{i}+\beta_{R} \bar{r}+\beta_{S} \bar{s}\right)\right) \\
& +\left(\beta_{R} \bar{r}+\beta_{S} \bar{s}\right)\left(\bar{b} \eta k+\bar{b} \beta_{S}\left(\frac{\bar{s}}{\overline{\bar{r}}} \sum_{i=1}^{n} \alpha_{i}\right)+k\left(\frac{\bar{s}}{\bar{r}} \sum_{i=1}^{n} \alpha_{i}+\beta_{R} \bar{r}+\beta_{S} \bar{s}\right)\right) \\
& +\underbrace{\left(\frac{\bar{s}}{\bar{r}} \sum_{i=1}^{n} \alpha_{i}\right) \bar{b} \eta k+k \bar{b} \beta_{S}\left(\frac{\bar{s}}{\bar{r}} \sum_{i=1}^{n} \alpha_{i}\right)-k \bar{b}\left(\frac{\bar{s}}{\bar{r}} \sum_{i=1}^{n} \alpha_{i}\right)\left(\eta+\beta_{S}\right)}_{=0} .
\end{aligned}
$$

Therefore, it is obtained that $a_{1} a_{2}-a_{3}>0$ due to (2) and (22). From Theorem 3(n=3), $\operatorname{Re} \lambda_{3,1}, \operatorname{Re} \lambda_{3,2}$ and $\operatorname{Re} \lambda_{3,3}<0$. According to Theorem 4, The equilibrium point $E_{3}$ in $\Omega$ is LAS for the system (3), when it exists biological, that is, $B<A$. Hence, proof is completed.

The LAS conditions found for equilibrium points in (13) are summarized in the Table 1. 
Proposition 6 Let us denote by $\Gamma_{2}$ the LAS region of the equilibrium point $E_{2}$ in $\Omega$. In the same way, $\Gamma_{3}$ is for $E_{3}$. Then $\Gamma_{2} \cap \Gamma_{3}=\emptyset$.

Proof It can be clearly observed in Table 1.

The parameter values and it's references used for numerical study are given in Table 2.

Proposition 7 If $A<B$, then the equilibrium point $E_{2}=(0, B, B, 1, \ldots, 1)$ is globally asimptotically stable (GAS) in $\Omega$.

Proof Their solutions approach $a_{i}=1$ for $i=1,2, \ldots, n$, when last $n$ equations of system (3) are considered separately. Replacing $s=0$ and $a_{i}=1$ for $i=1,2, \ldots, n$ in system

Table 1 Existence and stability conditions of the equilibria of system (3)

\begin{tabular}{lll}
\hline Equilibrium points & Biological existence conditions & LAS conditions \\
\hline$E_{0}=(0,0,0,1, \ldots, 1)$ & Always exists & Unstable \\
$E_{1}=(0,1,0,1, \ldots, 1)$ & Always exists & Unstable \\
$E_{2}=(0, B, B, 1, \ldots, 1)$ & Always exists & $A<B$ \\
$E_{3}=\left(A \frac{A-B}{A-B+C}, A \frac{C}{A-B+C}, A, 1,1, \ldots, 1\right)$ & $B<A$ & When it exists biological \\
\hline Where the values $A, B$ and $C$ are as indicated in (9) &
\end{tabular}

Table 2 Interpretation and considered values of the parameters

\begin{tabular}{|c|c|c|c|}
\hline Parameter & Description & Value & References \\
\hline$\beta_{S}$ & Growth rate of sensitive bacteria & 0.8 day $^{-1}$ & Mondragón et al. (2014) \\
\hline $\begin{array}{l}(1-c) \beta_{S} \\
0<c<1\end{array}$ & Growth rate of resistant bacteria & $0.4-0.1$ day $^{-1}$ & $\begin{array}{l}\text { Mondragón et al. } \\
\text { (2014)-hypothesis }\end{array}$ \\
\hline k & Growth rate of immune cells & 0.6 day $^{-1}$ & $\begin{array}{l}\text { Pugliese and Gandolfi } \\
\text { (2008) }\end{array}$ \\
\hline$\eta$ & $\begin{array}{l}\text { Rate of bacteria destroyed by } \\
\text { immune cells }\end{array}$ & 0.3 day $^{-1}$ & $\begin{array}{l}\text { Pugliese and Gandolfi } \\
\text { (2008) }\end{array}$ \\
\hline$\omega$ & $\begin{array}{l}\text { Rate to the amount of present bacteria } \\
\text { of carrying capacity of immune cells }\end{array}$ & 1 & Hypothesis \\
\hline$T$ & Carrying capacity of bacteria & $10^{9}$ bacteria & Alavez et al. (2006) \\
\hline$\overline{\alpha_{1}}$ & Mutation rate of INH & $10^{-6}$ mut $\times$ gen & Coll (2009) \\
\hline$\overline{\alpha_{2}}$ & Mutation rate of $\mathrm{PZA}$ & 0 & Mondragón et al. (2014) \\
\hline$\overline{d_{1}}$ & $\begin{array}{l}\text { Elimination rate of sensitive bacteria } \\
\text { due } \mathrm{INH}\end{array}$ & 0.0039 day $^{-1}$ & Zhang (2009) \\
\hline$\overline{d_{2}}$ & $\begin{array}{l}\text { Elimination rate of sensitive bacteria } \\
\text { due PZA }\end{array}$ & 0.0001625 day $^{-1}$ & Alavez et al. (2006) \\
\hline$\delta_{1}$ & Daily dose of INH & $5 \mathrm{mg} / \mathrm{kg} / \mathrm{day}$ & Coll (2009) \\
\hline$\delta_{2}$ & Daily dose of ZPA & 35-20 mg/kg/day & Coll (2009) \\
\hline$\mu_{1}$ & Uptake rate of INH & 0.06 day $^{-1}$ & Esteva et al. (2011) \\
\hline$\mu_{2}$ & Uptake rate of PZA & 0.03 day $^{-1}$ & Esteva et al. (2011) \\
\hline
\end{tabular}

Datas are deduced from the literature

The values obtained from this table are that (i) in the first case, $A=0.260674, B=0.571428(A<B)$ and so, $E_{2}=(0,0.5714,0.5714,1, \ldots, 1)$ is LAS. (ii) in the second case, $A=0.334538, B=0.25(A>B)$ and so, $E_{3}=(0.3337,0.0008,0.3345,1,1, \ldots, 1)$ is LAS

Among the treatment regimen recommended by WHO includes isoniazid (INH) and pyrazinamide (PZA) for some bacterial infectious (such as Mycobacterium tuberculosis) (Coll 2009) 
(3), we attain the asymptotically equivalent planar system (Mondragón et al. 2014) in the region $\Omega_{1}=\left\{(r, b) \in R^{2}: 0<b \leq r \leq 1\right\}$ given by

$$
\begin{aligned}
& \frac{d r}{d t}=f(r, b)=\beta_{R} r(1-r)-\eta r b \\
& \frac{d b}{d t}=g(r, b)=k b\left(1-\frac{b}{r}\right)
\end{aligned}
$$

According to the Dulac criterion, there exists a continuously differentiable Dulac function $\Phi(r, b)$ for a simply connected region $\Omega_{1} \subset R^{2}$ such that

$$
\Phi(r, b)=\frac{1}{r b} .
$$

Since $(f(r, b), g(r, b))$ is the vector field of system (26),

$$
\begin{aligned}
& \frac{\partial[\Phi(r, b) f(r, b)]}{\partial r}+\frac{\partial[\Phi(r, b) g(r, b)]}{\partial b}=\frac{\partial}{\partial r}\left[\frac{\beta_{R}(1-r)-\eta b}{b}\right]+\frac{\partial}{\partial b}\left[\frac{k\left(1-\frac{b}{r}\right)}{r}\right] \\
& =-\left(\frac{\beta_{R}}{b}+\frac{k}{r^{2}}\right)<0 .
\end{aligned}
$$

This result reveals that system (26) has no periodic orbits contained in the interior of $\Omega_{1}$ in compliance with Dulac-Bendixon criterion. In addition, the region $\Gamma_{2}$ cited in the Proposition 6 does not include another the LAS equilibrium point. Thus, by the Poincaré-Bendixon Theorem and Dulac-Bendixon criterion, we have that equilibrium point $E_{2}=(0, B, B, 1, \ldots, 1)$ is GAS.

For status (i) obtained from the Table 2, the qualitative analysis of the system (2) has supported by numerical simulations in the following Figs. 1, 2 and 3. Here, it is shown that the equilibrium point $E_{2}$ is GAS. Also, as a result of using multiple antibiotics at least for 60 days, sensitive bacteria is removed and resistant bacteria and immune cells have same equilibria value of $\frac{\beta_{R}}{\beta_{R}+\eta}$.

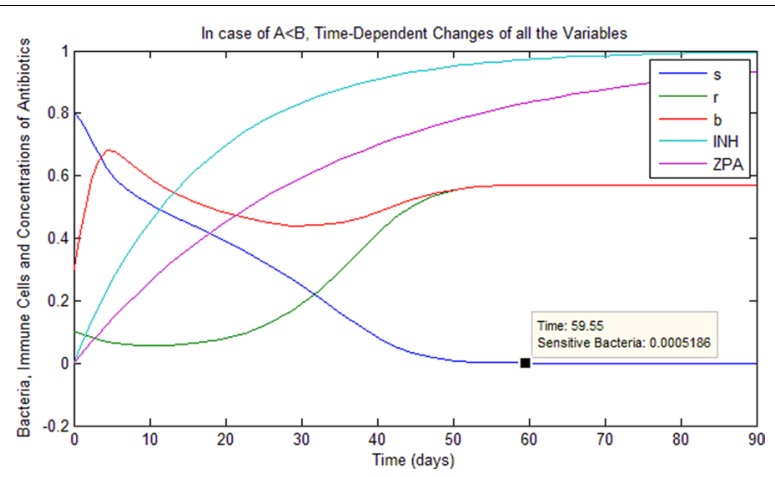

Fig. 1 In case of (i) in the Table 2, time-dependent changes of all the variables 


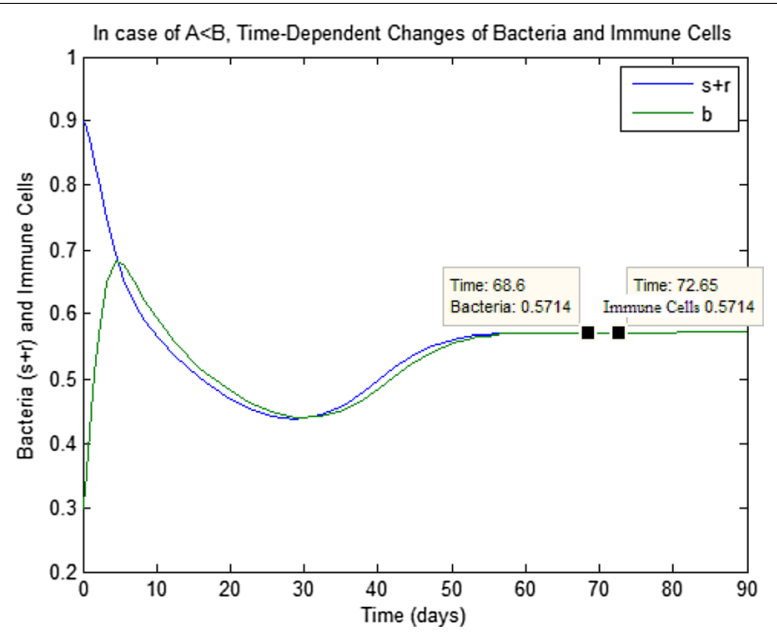

Fig. 2 In case of (i) in the Table 2, time-dependent changes of bacteria and immune cells

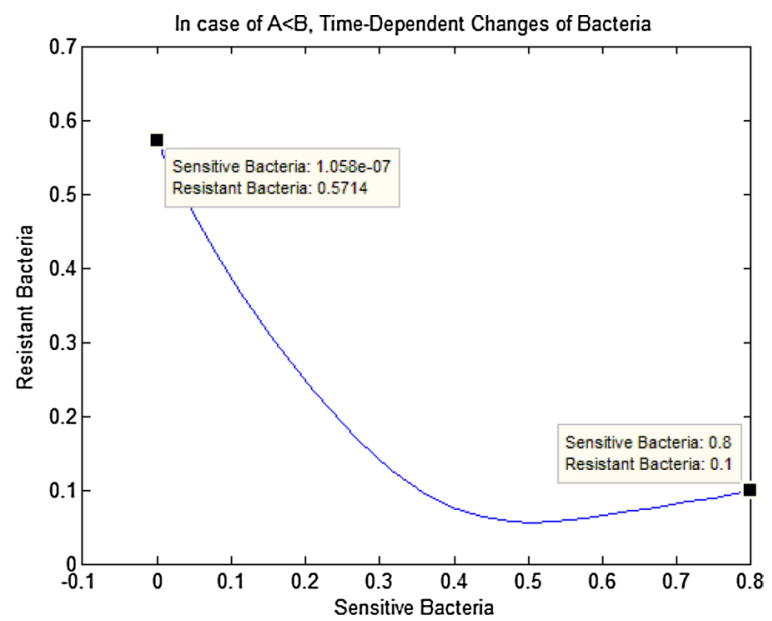

Fig. 3 In case of (i) in the Table 2, time-dependent changes of bacteria

Proposition 8 Let $B<A$. Then,

$$
\frac{b-\bar{b}}{b} \frac{d b}{d t}<0
$$

where the values $\bar{s}, \bar{r}$ and $\bar{b}$ are in (22).

Proof Let $b<\bar{b}$. This shows that $b$ is an increasing function and approach asymptotically to $\bar{b}$ by increasing in $\Omega$. From the third equation in system (3), it is obtained that $\frac{d b}{d t}=k b\left(1-\frac{b}{s+r}\right)>0$ except for $\bar{b}=\bar{s}+\bar{r}$ (state of equilibrium). In this sense, we have that $\frac{b-\bar{b}}{b} \frac{d b}{d t}<0$.

Let $b>\bar{b}$. In the same way, $b$ is approaches asymptotically to $\bar{b}$ by decreasing. Thus, it is obtained that $\frac{b-\bar{b}}{b} \frac{d b}{d t}<0$. Proposition is proved. 
Proposition 9 Let $A>B$. If $1-\bar{r}<s$ and $\frac{A-(1-\sigma) b}{\sigma}<s+r<\frac{A-(1-B) b}{B}$, then equilibrium point $E_{3}$ is $G A S$ in the region

$$
\Omega_{2}=\left\{(s, r, b) \in R^{3}: 0<b \leq s+r \leq 1\right\} .
$$

where $\sigma=\frac{\beta_{S}}{\beta_{S}+\eta}$.

Proof If the last $n$ equations of system (3) are separated, then their solutions approach $a_{i}=1$ for $i=1,2, \ldots, n$. Replacing these values in the first three equations of system (3), we attain the following:

$$
\begin{aligned}
\frac{d s}{d t} & =\beta_{S} s(1-(s+r))-\eta s b-s \sum_{i=1}^{n}\left(\alpha_{i}+d_{i}\right) \\
\frac{d r}{d t} & =\beta_{R} r(1-(s+r))-\eta r b+s \sum_{i=1}^{n} \alpha_{i} \\
\frac{d b}{d t} & =k b\left(1-\frac{b}{s+r}\right) .
\end{aligned}
$$

in the region (29). Considering (9), then the system (30) transforms to

$$
\begin{aligned}
\frac{d s}{d t} & =\left(\beta_{S}+\eta\right) s(A-(s+r) \sigma-b(1-\sigma)) \\
\frac{d r}{d t} & =\left(\beta_{R}+\eta\right)(r(1-(s+r)) B-r b(1-B)+s C) \\
\frac{d b}{d t} & =k b\left(1-\frac{b}{s+r}\right) \\
\text { where } \sigma & =\frac{\beta_{S}}{\beta_{S}+\eta} . \text { Also, it is clear that } \sigma>A .
\end{aligned}
$$

In this context, the GAS of equilibrium point $E_{3}$ can be obtained by applying the LaSalle-Lyapunov Theorem. Lyapunov function is

$$
V(s, r, b)=\left(c_{1}\left(s-\bar{s}-\bar{s} \ln \frac{s}{\overline{\bar{s}}}\right)+c_{2}\left(r-\bar{r}-\bar{r} \ln \frac{r}{\overline{\bar{r}}}\right)+c_{3}\left(b-\bar{b}-\bar{b} \ln \frac{b}{\bar{b}}\right)\right)
$$

where $c_{1}, c_{2}$ and $c_{3}$ are arbitrary positive constants and $\bar{s}, \bar{r}$ and $\bar{b}$ are in (22). Differentiating $V(s, r, b)$ with respect to $t$, we get

$$
\frac{d V}{d t}=c_{1} \frac{s-\bar{s}}{s} \frac{d s}{d t}+c_{2} \frac{r-\bar{r}}{r} \frac{d r}{d t}+c_{3} \frac{b-\bar{b}}{b} \frac{d b}{d t}
$$

Let $1-\bar{r}<s$. In this case, since $0<1-(\bar{s}+\bar{r})<s-\bar{s}$ and $1-\bar{r}+r<s+r<1$, it is obtained that

$$
\bar{s}<s, \quad \bar{r}>r,
$$

respectively. In addition, if $\frac{A-(1-\sigma) b}{\sigma}<(s+r)<\frac{B-(1-B) b}{B}$, then

$$
\frac{d s}{d t}<0, \quad \frac{d r}{d t}>0
$$


By (34) and (35), we have

$$
\frac{s-\bar{s}}{s} \frac{d s}{d t}<0, \quad \frac{r-\bar{r}}{r} \frac{d r}{d t}<0 .
$$

From (28) and (36), It is obtained that $\frac{d V}{d t}<0$. In this respect, if $1-\bar{r}<s$ and $\frac{A-(1-\sigma) b}{\sigma}<s+r<\frac{B-(1-B) b}{B}$, then equilibrium point $E_{3}$ is GAS for system (31) in $\Omega_{2}$, and so, for system (3) in $\Omega$. Proposition is proved.

For status (ii) obtained from the Table 2, the qualitative analysis of the system (3) has supported by numerical simulations in Fig. 4 . In this case, it is found that $E_{3}$ is GAS. Moreover, sensitive and resistant bacteria to multiple antibiotics and immune system cells have positive equilibrium values as in $E_{3}$.

\section{Conclusions}

In this paper, we formulated a mathematical model of bacterial resistance to immune system response and multiple antibiotics simultaneously, considering specific changes in bacterial DNA sequence as the only mechanism of bacterial resistance acquisition in order to evaluate the effectiveness of antibiotic treatments with respect to the mechanism above.

The parameter $A$ is interpreted as the number of bacteria produced by the fraction of sensitive bacteria that survive to the effects due to antibiotics and immune cells. Similarly, the parameter $B$ represents the bacteria produced by resistant bacteria. Also, the parameter $\eta$ is expresses as effect of immune cells on the bacteria .

Our model is quite appropriate when compared to the complexity of biological phenomenon and it predicts in terms of the parameters $A$ and $B$ when the bacterial progression is either for resistant bacteria and immune cellsas shown in Figs. 1, 2 and 3 or for sensitive and resistant bacteria and immune cells as shown in Fig. 4.

The model suggests that if sensitive bacteria can infect but do not produce sufficient progeny (in case of $A<B$ ) then they can be removed and resistant bacteria continue to survive in balance with the immune cells in the host. When sensitive bacteria persist, the model predicts the scenario that the immune response of host and antibiotics is not

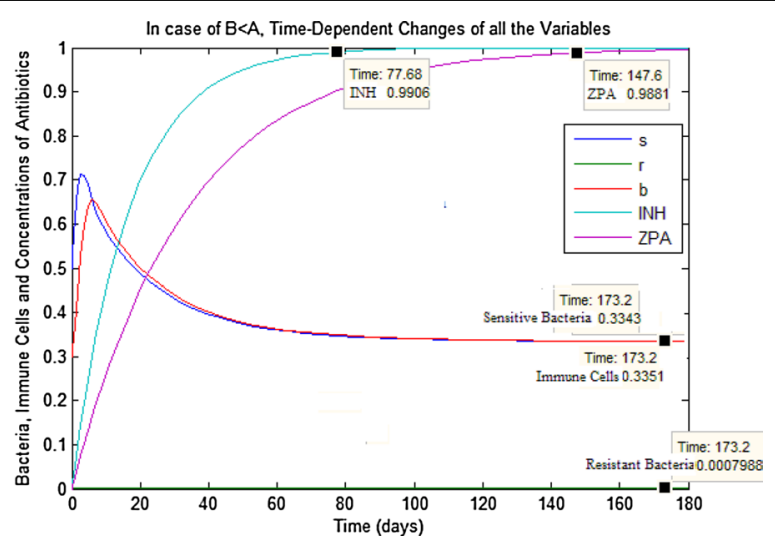

Fig. 4 In case of (ii) in the Table 2, time-dependent changes of all the variables 
enough to eliminate them (in case of $A>B$ ), and therefore both types of bacteria continue to survive and coexist in balance with the immune cells in the host.

According to the results of this analysis, the infection never disappears. Also, the infection is continued by resistant bacteria, when the appropriate antibiotics are used, otherwise sensitive and resistant bacteria. The magnitude of infection depends on the effect of immune system in the first case and multiple antibiotics and immune system in the second case.

These results in our model highlight the fact that those whose immunity response against infections have diminished, suffer from the same bacterial infections more. Furthermore, this model shows that some of the bacterial infections believed to be limited or destroyed, make an individual whose immune system deteriorated suffer more.

Additionally, the results obtained from numerical studies in terms of bacterial infection reveal the affinity substantially with reference to the clinical treatment.

For future work we are planing consider other mechanisms such as the loss of resistance in the resistant bacteria and gaining resistance by conjugation of sensitive and resistant bacteria in order to get more accurate results.

Authors' contributions

Both authors have contributed to the manuscript equally. Both authors read and approved the final manuscript.

\section{Author details}

${ }^{1}$ Department of Computer Technologies, Kangal Vocational High-Schools, Cumhuriyet University, 58840 Sivas, Turkey.

2 Department of Mathematics, Faculty of Sciences, Erciyes University, 38039 Kayseri, Turkey.

\section{Competing interests}

The authors declare that they have no competing interests.

Received: 16 November 2015 Accepted: 16 March 2016

Published online: 05 April 2016

References

Alavez J, Avenda R, Esteva L et al (2006) Within-host population dynamics of antibiotic-resistant M. tuberculosis. Math Med Biol 24:35-56

Alberts B, Johnson A, Lewis J et al (2002) Molecular biology of the cell. In: The adaptive immune system, 4th edn. Garland Science, New York. ISBN 10: 0-8153-3218-1

Allen LJS (2007) An introduction to mathematical biology. ISBN 10: 0-13-035216-0

Andersson DI, Levin BR (1999) The biological cost of antibiotic resistance. Curr Opin Microbiol 2:489-493

Andersson DI, Björkholm B, Sjölund M et al (2001) Mutation frequency and biological cost of antibiotic resistance in Helicobacterpylori. Proc Natl Acad Sci 98(25):14607-14612

André JB, Gandon S (2006) Vaccination, within-host dynamics, and virulence evolution. Evolution 60(1):13-23

Antia R, Koella JC, Perrot V (1996) Models of within-host dynamics of persistent mycobacterial infections. Proc R Soc Lond B 263:257-263

Arya DP (2007) Aminoglycoside antibiotics: from chemical biology to drug discovery. Wiley, New Jersey

Austin D, Anderson R (1999) Studies of antibiotic resistance within the patient, hospitals and the community using simple mathematical models. Philos Trans R Soc Lond B 354(1384):721-738

Austin D, Kakehashi M, Anderson RM (1997) The transmission dynamics of antibiotic-resistant bacteria: the relationship between resistance in commensal organisms and antibiotic consumption. Proc R Soc Lond B 264(1388):1629-1638

Bonhoeffer S, Lipsitch M, Levin BR (1997) Evaluating treatment protocols to prevent antibiotic resistance. Proc Natl Acad Sci USA 94(22):12106-12111

Bonten M, Austin J, Lipsitch M (2001) Understanding the spread of antibiotic resistant pathogens in hospitals, mathematical models as tools for control. Clin Infect Dis 33(10):1739-1746

Bootsma MCJ, Van Der Horrst MA, Guryeva T et al (2012) Modeling non-inherited antibiotic resistance. Bull Math Biol 74:1691-1705

Butler MS, Buss AD (2006) Natural products—-the future scaffolds for novel antibiotics? Biochem Pharmacol 71(7):919-929

Carvalho RV, Kleijn J, Meijer A et al (2012) Modeling innate immuneresponse to early mycobacterium infection. Comput Math Methods Med 790482:1-12

Clatworthy AE, Pierson EP, Hung DT (2007) Targeting virulence: a new paradigm for antimicrobial therapy. Nat Chem Biol 3:541-548

Coll P (2009) Fármacos con actividad frente a Mycobacterium tuberculosis. Enferm Infecc Microbiol Clin 27(8):474-480 
D'Agata E, Magal P, Olivier D et al (2007) Modeling antibiotic resistance in hospitals: the impact of minimizing treatment duration. J Theor Biol 249:487-499

D'Onofrio A (2005) A general framework for modeling tumor-immune system competition and immunotherapy: mathematical analysis and biomedical inferences. Phys D 208:220-235

Esteva L, Mondragon El, Leyton JR (2011) Un modelo matemático sobre bacterias sensibles y resistentes a antibióticos. Mat Enseñ Univ 19(2):55-73

Gilchrist M, Coombs D (2006) Evolution of virulence: interdependence, constraints and selection using nested models. Theor Popul Biol 69:145-153

Gilchrist M, Sasaki A (2002) Modeling host-parasite coevolution: a nested approach based on mechanistic models. J Theor Biol 218:289-308

Haber M, Levin BR, Kramarz P (2010) Antibiotic control of antibiotic resistance in hospitals: a simulation study. BMC Infect Dis. doi:10.1186/1471-2334-10-254

Hethcote HW (2000) The mathematics of infectious diseases. SIAM Rev 42:599-653

Johnson PJT, Levin BR (2013) Pharmacodynamics, population dynamics and the evolution of persistence in Staphyloccus aureus. PLoS Genet 9(1):1-13

Kostova T (2007) Persistence of viral infections on the population level explained by an immunoepidemiological model. Math Biosci 206(2):309-319

Lewis K (2013) Platforms for antibiotic discovery. Nat Rev Drug Discov 12:371-387

Linares J, Martinez J (2005) Resistencia a los antimicrobianos y virulencia bacteriana. Enferm Infecc Microbiol Clin 23(2):86-93

Mahmoud AG, Rice LB (1999) Antifungal agents: mode of action, mechanisms of resistance, and correlation of these mechanisms with bacterial resistance, and correlation. Clin Microbiol Rev 12(4):501-517

Massad E, Nascimiento M, Bezerra F (2008) An optimization model for antibiotic use. Appl Math Comput 201:161-167

Mohtashemi M, Levins R (2001) Transient dynamics and early diagnosis in infectious disease. J Math Biol 43:446-470

Mondragón El, Mosquera S, Cerón M et al (2014) Mathematical modeling on bacterial resistance to multiple antibiotics caused by spontaneous mutations. BioSystems 117:60-67

Nowak MA, May R (2000) Virus dynamics: mathematical principles of immunology and virology. Oxford University Press, Oxford

Opatowski L, Guillemot D, Bolle PY et al (2011) Contribution of mathematical modeling to the fight against bacterial antibiotic resistance. Curr Opin Infect Dis 24(3):279-287

Pugliese A, Gandolfi A (2008) A simple model of pathogen-immune dynamics including specific and non-specific immunity. Math Biosci 214:73-80

Rodriques P, Gomes M, Rebeloc C (2007) Drug resistance in tuberculosis—a reinfection model. Theor Popul Biol 71:196-212

Singer B (1984) Mathematical Models of infectious diseases: seeking new tools for planning and evaluating contro programs. Suppl Popul Dev Rev 10:347-365

Sotto A, Lavigne JP (2012) A mathematical model to guide antibiotic treatment strategies. BMC Med. doi:10.1186/1741-7015-10-90

Sun HR, Lu X, Ruan S (2010) Qualitative analysis of models with different treatment protocols to prevent antibiotic resistance. Math Biosci 227:56-67

Ternent L, Dyson RJ, Krachler A-M et al (2014) Bacterial fitness shapes the population dynamics of antibiotic resistant and susceptible bacteria in a model. J Theor Biol 372:1-11

Whitman A, Ashrafiuon H (2006) Asymptotic theory of an infectious disease model. J Math Biol 53(2):287-304

Wiesch PA, Kouyos R, Engelstadter J et al (2011) Population biological principles of drug- resistance evolution in infectious diseases. Lancet Infect Dis 11(3):236-247

Zhang Y (2009) Mechanisms of drug resistance in Mycobacterium tuberculosis. Int J Tuberc Lung Dis 13(11):1320-1330

\section{Submit your manuscript to a SpringerOpen ${ }^{\circ}$ journal and benefit from:}

- Convenient online submission

- Rigorous peer review

- Immediate publication on acceptance

- Open access: articles freely available online

- High visibility within the field

- Retaining the copyright to your article

Submit your next manuscript at $\boldsymbol{s p r i n g e r o p e n . c o m ~}$ 